\title{
RESEARCH PAPER \\ MANUFACTURING ENGINEERING PRACTICES IN THE METAL FABRICATIONS SECTOR OF GHANAIAN INDUSTRY
}

\author{
S. M. Sackey ${ }^{1}$ and S. A. Frimpong ${ }^{2}$ \\ ${ }^{\text {I} M e c h a n i c a l ~ E n g i n e e r i n g ~ D e p a r t m e n t, ~ K ~ N U S T, ~ K u m a s i ~}$ \\ ${ }^{2}$ Mechanical Engineering Department, Kumasi Polytechnic, Ghana \\ Corresponding author: sackeysm@yahoo.com
}

\begin{abstract}
The purpose of this paper is to find out manufacturing engineering (ME) practices in the metals sector of the Ghanaian industry. The paucity of such works in the literature means that the real state of ME practice is largely unknown, despite the fact that it remains an indispensable support function to industry. ME involves design and specification of tooling, production processes, process planning and technical problemsolving. Following ME principles make it far more likely that products will meet their design specifications and made within the estimated cost and time. In this study the practice of $M E$ is investigated using a questionnaire sent to 50 sampled metal processing firms in three major cities but returned by 25 of them. Thirteen of them are from Kumasi, 5 from Tema, and 7 from Accra. Data gathered were analysed using descriptive statistics including graphs. Comparisons were also made with global best practice found in the literature. The results suggest that the classical functions of $M E$ are being practiced to various degrees in the firms studied. However, production facilities employed are mostly of the conventional type and equipment such as computer numerical control machines (CNCs), industrial robots, automated material handling systems, and flexible manufacturing systems are not in place. By this absence, many advantages of CNC and other automated production machine tools such as higher precision and repeatability, greater flexibility, reduced cutting and idle times etc., are missed by metal processing firms in Ghana. Further, firms do not use any computer systems for design/manufacturing, a situation that can only be bad for competitiveness and growth, because computer aided design (CAD) and manufacturing systems, including computer aided process planning, accelerate the design process to integrate it with manufacturing by eliminating artificial barriers that lead to long lead-times. In addition $40 \%$ of respondent firms retain manufacturing engineers who do not contribute to Design for Manufacture (DFM) or Design for Assembly. This confirms a major factor responsible for a reported low rate of new product introduction; moreover, where manufacturing engineers do offer some contribution to DFM, they do not consciously have product innovation in mind as a target goal, a situation which can best be described as unprogressive.
\end{abstract}

Keywords: Metal processing, manufacturing engineering, practices, Ghana

\section{INTRODUCTION}

Research purpose and importance of manufacturing engineering

The aim of this paper is to assess the status of manufacturing engineering (ME) practices in industries in Ghana involved in metal fabrications to help in understanding what is currently happening so that recommendations and strategies could be developed to improve industries. The intent is to investigate those practices pri- 
marily in light of classical best practice and also, to a lesser extent, in light of current global state-of-the-art developments. Research works focusing on ME are rare in the Ghanaian literature, and its current level of development in the country is largely unknown; yet, by its nature, ME remains an indispensable support function to industry.

Classical manufacturing engineering involves process planning and tooling design as its core elements. Other functions include designing individual production processes, specifying new production facilities and materials handling devices and systems, shop-floor problem solving, monitoring production processes, inspecting finished products, and quality control and assurance checkpoints. These as are supported by Deming's (2000) framework for quality and productivity improvement, which promotes continuous improvement and a get-itright-the-first-time mind-set. Manufacturing engineering further involves, as well, responsibility for working with, and giving advice to product designers on product production . Applying the above principles make it far more likely that a product will meet the original design specifications and could be made in time at the estimated cost.

Investigating these concepts in the metal fabrications sector of Ghanaian industry could help bring to light the extent to which this vital support function is being performed and identify inherent problems relating to the discipline. This, in turn, could help inspire the needed actions towards improved performance.

\section{State of the art in global manufacturing en- gineering}

The purpose of this section is to highlight technology issues impacting the nature and practice of manufacturing engineering in the industrialised world in order to aid benchmarking comparisons in later sections of this article with Ghana.

Two keywords that probably best describe trends in manufacturing engineering practice in an industrialised country and that set it apart from that most likely to be found in a developing country, such as Ghana, are: automation and integration, i.e. manufacturing's automation and integration with the design function. Thus in modern global manufacturing engineering practice common concepts include: (1) Computer Aided Process Planning (CAPP), which is the determination of processes and resources needed for completing the manufacturing processes required for converting raw materials into a final product to satisfy the design requirements and intent while respecting the geometric and technological constraints of the design (Scallan, 2003); (2) CADCAM, Robotics and Manufacturing Automation and (3) Integrated Product and Process Design and Manufacturing (Gunasekaran and Yusuf, 2002) where in the evolution of product design and manufacture, manufacturing issues involved in multi-product realisation are addressed early-on (Sheheryar and Chang, 2014) by integration of design and manufacturing processes (Xue-Feng et al, 2013), taking cognizance of the interfacial link between design for manufacture (DFM) and design for assembly (DFA). Ulrich and Ellison (2009) have noted, however, that the interaction between product design and manufacturing engineering is yet to be fully explored from an integral perspective; (4) Flexible Manufacturing Systems (Xu et al, 2013), in which enterprises can create product and manufacturing diversity through component flexibility, control flexibility, mixed flexibility and modularity design (Baldwin and Kim, 2000); (5) Computer Numeric Control Manufacturing; (6) 3D-Printing, which is a common name given to a host of related technologies including rapid prototyping, stereolithography used to fabricate physical objects directly from CAD data sources (Oppon et al., 2014). Such systems are also known by the names: additive manufacturing, layered manufacturing and many others. Additive technologies offer other advantages as compared to classical subtractive fabrication methods such as milling or turning. These include the ability to form items with 
any geometric complexity, and speed.

It is clear from the foregoing, that the manufacturing engineering requirements of these modern systems are clearly different from those of the traditional manufacturing engineering function. Whereas in traditional systems these functions are clearly defined and more regimented, in modern systems there is indeed greater automation and integration of the manufacturing and design functions. Thus a basis has been prepared for benchmarking local practices (to be presented and discussed), with these modern systems.

\section{Research Aim and Objectives}

This work aims to assess the status of manufacturing engineering in the metal processing sector of Ghanaian industry. The objectives are to:

- Investigate the application of manufacturing engineering principles in the metal processing sector of Ghanaian industry primarily in light of classical best practice, and to a lesser extent, current global stateof-the-art developments impacting the discipline,

- Explore the general level of technology and automation employed by firms in the metal processing sector of the industry.

- Identify technical manpower issues relating to the practice of manufacturing engineering in Ghana.

- Identify challenges which hamper the application of these principles in the sector so that recommendations and strategies could be developed to improve industries.

\section{RESEARCH METHODOLOGY} Population, sampling procedures and sample size

\section{Population}

Since most industrial activity in Ghana is concentrated in Accra, Tema and Kumasi, the industries selected for the study were drawn from those three centres. Manufacturing firms under the Association of Ghana Industries member ship register of the year 2011 (AGI, 2011) constituted the total (aggregate) population (237) at the disposal of the study. By city this breaks down as: 132 firms from Accra, 61 firms from Tema, and 44 from Kumasi. Information as to the proportions of these involved in metal processing was not available. But if we assumed a third of firms were involved in metal processing, then this would translate roughly to 44 firms in Accra, 20 in Tema, and 15 from $\mathrm{Ku}-$ masi, giving the total population of metal processing firms as 79 .

\section{Sampling procedure and sample size}

Sampling units employed in the survey include the categories of the National Board for SmallScale Industries (NBSSI) in which firms with less than 6 staff are classified as micro, firms with between 6 and 29 staff are designated small, firms with 30 to 49 staff are called medium, and firms with more than 50 staff are designated large. A desire to have a broad based spectrum of sampling units, led to the inclusion in the survey of a much larger industrial concern (a food and beverage can manufacturer) outside the NBSSI's categories. In addition five large firms, all situated in Accra or Tema, were surveyed. The rest are either small or medium in size.

Fifty firms were sampled from the total of 79 metal processing firms but only twenty five responded favourably to the questionnaire, thirteen (13) of them coming from Kumasi, five (5) from Tema, and seven (7) from Accra. This represents a response rate of $50 \%$ and close to $(25 / 79) \times 100=32 \%$ of the total population. By the NBSS criteria, 6 firms are large, 10 are medium, 6 are small, and 2 are micro in size. All the large and larger firms are from the formal sector of industry. Further, the companies selected represent a cross section of metal products, enabling the researchers to test the principles of ME across firms of various sizes and with different concentrations. 
42

\section{Research Instrument}

A survey method was used to gather data on manufacturing engineering practices in selected metal processing industries in Ghana. Data was collected using a structured questionnaire administered personally at the premises of the surveyed firms. In most cases the questionnaire was left with the firm and the response collected at a later date, when a meeting was held with personnel in charge of the manufacturing processes, to offer any clarifications, if needed.

The nature of the questionnaire used was such that it sought to address issues, themes or practices of interest such as: product mix and rate of new product introduction as an indication of innovative capability; use of engineering drawings as a pointer to the extent to which quality and standard engineering protocols were being followed; technology employed, to help reveal the general skills and level of manufacturing engineering called for; shop-floor technical problem solving, as a measure of depth of involvement of manufacturing engineers in ensuring smooth production and continuous improvement; tool design, to ascertain whether standard protocols and infrastructure such as tool rooms and standardised inputs were in place; process planning and its level of automation and integration with the design function, as the most fundamental function of manufacturing engineering.

\section{Data Analysis}

The statistical software tools SPSS and STATA were used for data input and analysis. Each objective of the research was analyzed using appropriate statistical measures. Descriptive statistics was used to analyse the data and this included plotting of graphs. The standard practices in firms such as level of in-house making of production tooling, the use of engineering drawings, and the application of statistical quality control techniques and computer systems for design and manufacturing were described using frequency counts and percentages.

\section{RESULTS AND DISCUSSION \\ Product mix and quantities}

The first question in the questionnaire sought to find out the product mix and their quantities from metal processing industries. Sixty-six (66) products were recorded from all the twenty-five metal processing industries. These products are grouped into categories that reflect their end use and means of manufacturing as shown in Table 1.

Other products are mining and construction machinery, timber processing machines, and metal furniture products.

The significance of the products and their quantities is that they are produced in-house, an evidence that manufacturing is taking place. Creation of products occur through the transformation of raw materials into finished articles. The efficiency of the transformation process is dependent on the selection of the best process and its parameters to optimize the quality characteristics of the finished products.

\section{Evolution of product designs and introduc- tion of new product}

Of the 25 firms, only 1 introduced 2 new products yearly. This may suggest that competition is not so keen and as such market analysis surveys to identify consumer needs, which would trigger the process to satisfy such needs are not well in place, or it may be an indication that product sales are not so encouraging. This conclusion is drawn in light of the quantities produced monthly, which are quiet low for several of the product groups (see Table 1). The economic success of manufacturing companies depends on their ability to identify the needs of customers and to quickly create products that meet these needs. Achieving these goals principally entails the acquisition of manufacturing engineering techniques.

From the indicated rate of new product introduction it further comes out that innovations made on existing products appear to be the order, and are rather of the incremental type inv- 
Table 1: Distribution of metal products and their quantities among surveyed firms

\begin{tabular}{lccc}
\hline Product & No. of firms & Percentage of firms (\%) & Yearly quantity produced \\
\hline Processed food cans & 1 & 4 & 173000000 whole cans \\
Gas cylinder products & 1 & 4 & 1356000 whole cylinders \\
Formed products & 1 & 4 & 43896 whole product units \\
Roofing sheets & 6 & 24 & 3240 tons \\
Domestic wares & 1 & 4 & $633677 \mathrm{~kg}$ \\
Other metal fabrications & 15 & 60 & 996 whole product units \\
\hline
\end{tabular}

olving small changes to an existing design. This makes it quite difficult to understand why the rate of introduction of new products by the firms is so low since innovations on existing products bring about improvement or new versions of the original product. Perhaps practitioners do not consciously have this as a target goal, a situation that can be described as not progressive.

Sixty percent of the firms indicated that manufacturing engineers contribute to Design for Manufacture (DFM). Twenty six percent (26\%) of the 15 firms that make up the $60 \%$ contribute to Design for Assembly (DFA). Areas of contribution to product design by manufacturing engineers are given as redesign and modification of client's designs.

The industry stands to benefit from some strands of research focusing on state-of-the-art methods for new product development that incorporate manufacturing aspects such as: Concurrent Engineering (Choi et al., 2010; Chen et al., 2002), and DFA and Quality Function Deployment (Carnevalli and Miguel, 2008). Using DFM and DFA guidelines as a design strategy should bring about several design alternatives, which could lead to more improved versions of the original product. Thus since $40 \%$ of firms do not contribute either to DFM or DFA, it confirms a major factor leading to the suspected low rate of innovation. With this outcome, the generation of optimum designs (which comes with reduced costs and development times) cannot be assured to win the confidence of management to introduce new products. DFM and DFA require the use of concurrent engineering and multifunctional design teams that ensure quickest time to market because of a "right first time" design and a smooth transition into production.

\section{Production facilities}

Production facilities available at the industries surveyed are mostly conventional, but modern state-of-the-art equipment such as computer numerically control (CNC) machines, 3DPrinting systems and related technologies such as stereolithography, industrial robots, automated material handling systems, and flexible manufacturing systems (Xu et al, 2013), are not in place. These systems are used widely in industrialised countries in the manufacture of products. Only one firm in the survey has CNC machines. Manufacturers worldwide are becoming more and more involved in the production of technologically advanced products and in the use of high technology in the manufacturing process itself.

Therefore, by this absence, many advantages of CNC production machine tools and 3D-Printing systems are missed by the metal processing sector of Ghanaian manufacturing industry. These include, for CNCs, higher precision and repeatability, greater flexibility, globally standard parts, reduced real cutting time, minimized idle time, among others. Industrial robots for their part are capable of executing a variety of 


\section{$44 \quad$ Sackey and Frimpong}

jobs ranging from material handling to complex assembling tasks. However, concerns about jobs being lost due to the use of industrial robots could result in resistance from the labour movement, because under this regime, increasing numbers of technically skilled people will work in control rooms rather than on the shop floor. For 3D-Printing systems these advantages include the ability to form objects with any geometric complexity or intricacy without the need for elaborate machine setup or final assembly, and rapid prototyping systems that reduce the construction of complex objects and tooling to a manageable, straightforward, and relatively fast process, thus reducing time to market in manufacturing (Oppon et al., 2014). Overall, these state-of-the-art technologies bring about improved productivity and reduced manufacturing costs.

\section{Computer systems for design and manufac- turing}

Eighteen, (i.e. 72\%) metal processing firms do not use any computer systems for design and manufacturing. This is bad for competitiveness and growth. As noted in section 1.2, modern global manufacturing engineering practice employs systems such as: (1) Computer Aided Process Planning (Scallan, 2003), which act as the link between product design and manufacturing, automates the translation of product design information into the process steps and instructions to manufacture the product, thus integrating manufacturing with the design function (Sheheryar and Chang (2014), Xue-Feng, et al (2013)); (2) Computer-aided Design and Manufacturing (CADCAM), Robotics and general manufacturing automation (Gunasekaran and Yusuf, 2002).

CADCAM systems accelerate the design process by eliminating artificial barriers between the traditional product design and manufacturing functions. The traditional product development cycle, such as is done in Ghanaian firms, isolates the roles of the designer, the supplier, the manufacturing engineer, and the assembly worker, resulting in increased development and production time and costs in addition to compromising each part's performance.

To take advantage of the numerous benefits of soft and hardware technologies in the Ghanaian environment, numerical control systems could be retrofitted to existing conventional machine tools. Then, old machines would not become waste and operators can slowly get used to new technology though their skills would have to be upgraded for this to happen. In addition answers must be found to pertinent questions on whether the existing conventional machines would be able to withstand the faster operating speeds of NC machines, how backlash and other machine inaccuracies would be accounted for, and whether any major mechanical modifications would be involved.

\section{Use of engineering drawings}

In the absence of higher-end computer aided manufacturing systems such as CADCAM and its benefits, engineering drawing is a vital tool for communicating information in an engineering manufacturing set up. It is used for (1) product design, (2) product development, (3) component and part manufacturing and (4) assembly. One would therefore expect that all the 25 firms would respond that they always use engineering drawings. However, from the survey $16 \%$ of firms "never" use engineering drawings. $84 \%$ of the firms use engineering drawings for all the above four purposes. This percentage agrees with the sum of percentages of firms that use engineering drawing "sometimes" and "always", so in absolute terms the figure may not be as high as it appears. Without the use of engineering drawings one wonders how consideration and determination of the sizes, shapes and tolerances of product parts and assemblies could be communicated in the manufacturing set-up. This situation could impact negatively on inspection, quality control, maintenance, tolerances, fits and acceptable standards. To achieve quality products, and enhance development of concepts for new products the use of engineering drawings should be a core part of the product reali- 
sation process of manufacturing firms.

\section{Subcontracting of jobs}

Seventy six percent $(76 \%)$ of the surveyed firms reported that they subcontract jobs to outside firms (Fig. 1), for reasons such as (i) meeting deadlines for delivery of products to customers, (ii) when the job is of civil engineering nature, (iii) equipment break-downs and (iv) when a customized job is called for (using special materials). this reason the extensive subcontracting of $76 \%$ may be viewed as a high-risk strategy.

\section{Problem solving responsibility}

Whenever a product is in production, manufacturing problems invariably arise. The problems on the shop-floor are varied, but their solution may enhance quality. Finding the causes of such problems even though primarily the job of the manufacturing engineer, must involve everyone, particularly shop-floor workers. The results show that this approach to problem

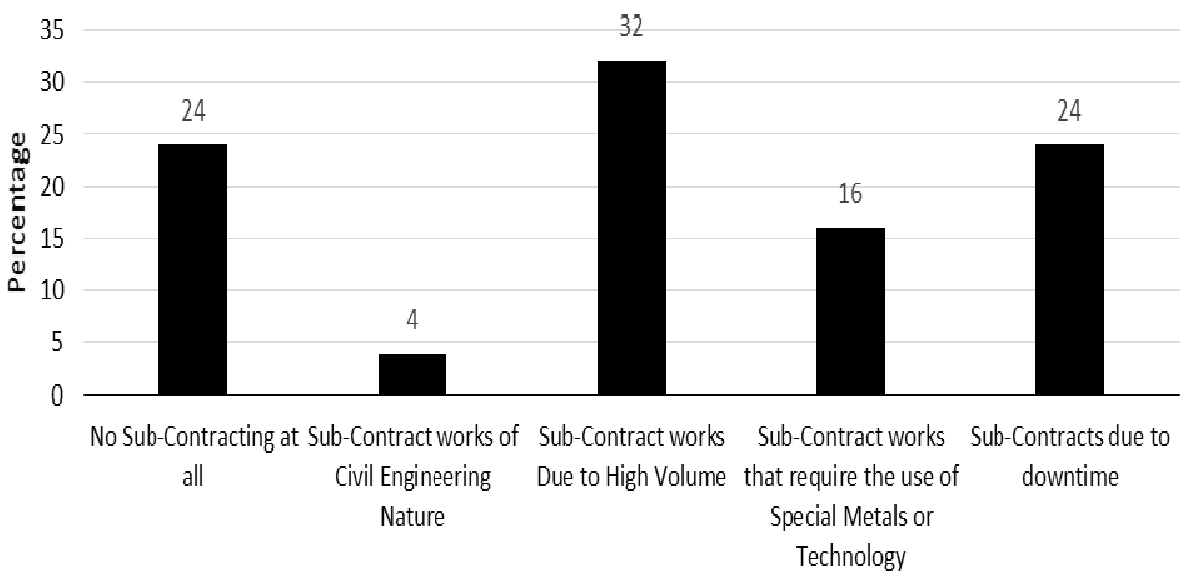

Firms

Fig. 1: Types of works that are sub-contracted

It is noted that subcontracting may interfere in several ways with a well-functioning in-house integrated product and process design and manufacturing programme (Gunasekaran and Yusuf, 2002) and unless special precaution are taken regarding smooth logistics, seamless communication, and general reliability, such as may not always be available in a developing country like Ghana, problems may arise. Thus, it is significant to note that whereas some level of subcontracting can be desirable to accommodate demand fluctuations, unless the relationship with the supplier (vendor, contractor) is particularly strong a manufacturer can lose control over schedule and quality. For solving is prevalent in the firms surveyed. Many workers on the shop-floor are involved in problem solving. This is a healthy sign that is gradually bringing improvements in the metal processing industries. When employees think that seeking out and suggesting improvements is the sole responsibility of specialists, they will stop looking for areas needing improvement. If they see a problem, they will not look for a solution, and if they have a solution, they will not tell anyone. The people doing the same tasks day-in and day-out often see improvement opportunities that experts overlook. 


\section{Staff training}

It is quite encouraging that $92 \%$ of firms do train their staff in manufacturing engineering related disciplines (Fig. 2). This suggests that these firms are serious about harnessing the power of their staff in order to be competitive progressively. $32 \%$ of the $92 \%$ train their staff yearly to upgrade skills, $24 \%$ pursue continuous education on the production line, $16 \%$ provide training on acquisition of new equipment with $20 \%$ of respondents training their staff only occasionally.

Nothing is static in today's world. Technology is changing rapidly. Training and educational programmes with appropriate resources and facilities are essential for achieving success in manufacturing. A manufacturing company becomes a winner over its competitors by attaining high performance with best practices and by sustaining it through the efforts of its staff. The success factor for attaining and sustaining high performance will be the quality of its staff.

\section{Development of process plans}

Process planning translates product design information into the process steps and instructions to manufacture the product efficiently and effectively. This very important manufacturing engineering function is used by $76 \%$ of the firms in their operations. However $76 \%$ of the selected firms do not use any computer system/software, or data sheets for design/ manufacturing, an indication that the development of the process plans is made manually. Manual process planning is based on a manufacturing engineer's experience and knowledge of production facilities, equipment, processes, and tooling and their capabilities. In this approach, parts are classified into families and standardized process plans are developed for these part families. Whenever a new part is called for, the process plan for that family would be manually retrieved, marked-up and retyped. this does not improve quality in planning, but rather may account for the low rate of new product introduction observed earlier because the process requires a lot of labour and high manufacturing lead-time. To introduce a new product means process planning activities such as interpretation of product design data, selection of manufacturing processes and machine tools, selection of cutting tools, determination of setup requirements, sequencing of operations, determination of the production tolerances etc., would have to be done manu-

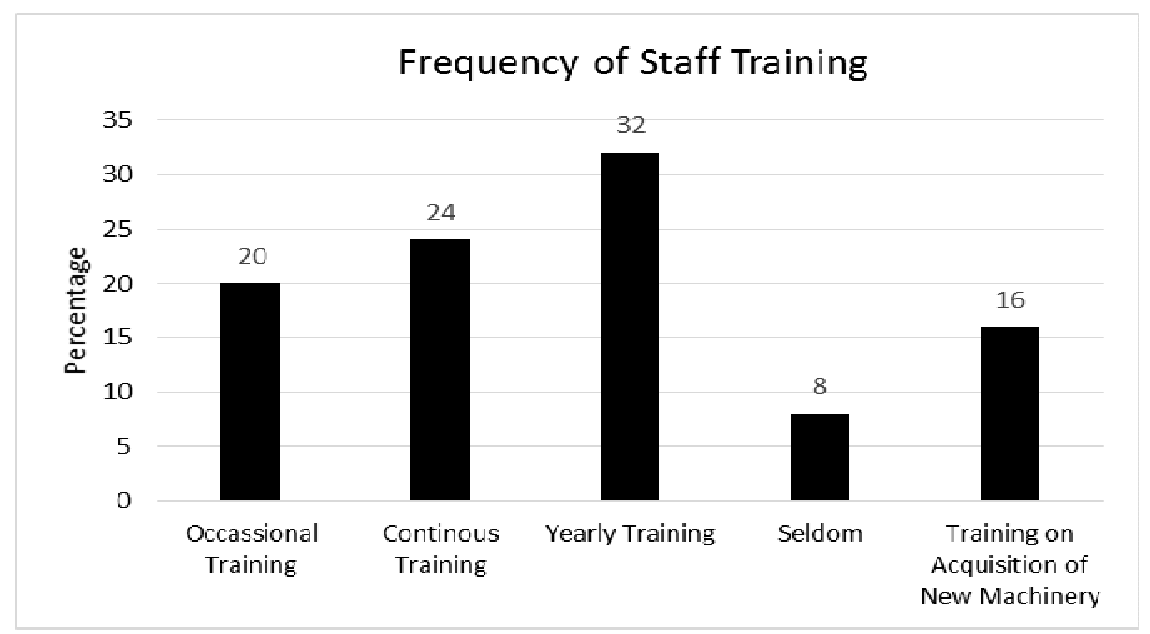

Fig. 2: Frequency of staff training in companies 
ally for all the parts of the product each time the need arises. This is the bane of the Ghanaian metal processing industry regarding the introduction of new products. Here, as noted under the section on state of the art in global manufacturing engineering, Ghanaian firms could benefit from state-of-the-art practices in modern process planning, namely computer aided process planning (Scallan, 2003) whose benefits include time and cost efficiencies, enhanced quality, easy and editable documentation, and product innovation potential, among others. However, the wide usage of manual process plans $(76 \%)$ in the study is a healthy indication that specifications of product parts, machining parameters, step-by-step work instructions, including dimensions related to individual operations, are in use to enhance product quality and facilitate manufacturing and assembly.

\section{Tooling design}

One of two key functions of manufacturing engineering is tool/tooling design, modification, and specification. From the responses, $92 \%$ of the firms design tooling in-house. The responsibility for this falls on Machinists (4 firms), Blacksmiths (1 firm), Technical Dir- ector (1 firm), Engineers/Supervisors (6 firms), Technical Department in consultation with user department (1 firm), Production Managers (2 firms), Workshop Managers/Sectional Heads (6 firms), with a range of qualifications such as B.Sc. Mechanical Engineering, Mechanical Engineering Technician Certificate and Basic Education Certificate. $84 \%$ of the firms surveyed produce jigs and fixtures frequently inhouse as well as Go- and No-Go gauges and material handling equipment. Significantly, this shows (Figs. 3 and 4) that at least, on the surface, a major manufacturing engineering function is being practiced in metal processing industries in Ghana, albeit at a somewhat low level.

The range of tooling designed in-house is limited (covering only inspection and gage design, and jig and fixture design). Other subjects such as materials used for tooling, die design, tool design for joining processes, and modern processes such as modular and automated tool handling, computer applications in tool design, geometric dimensioning and tolerancing (Nee, 2010), are known not to be in place in the firms surveyed.

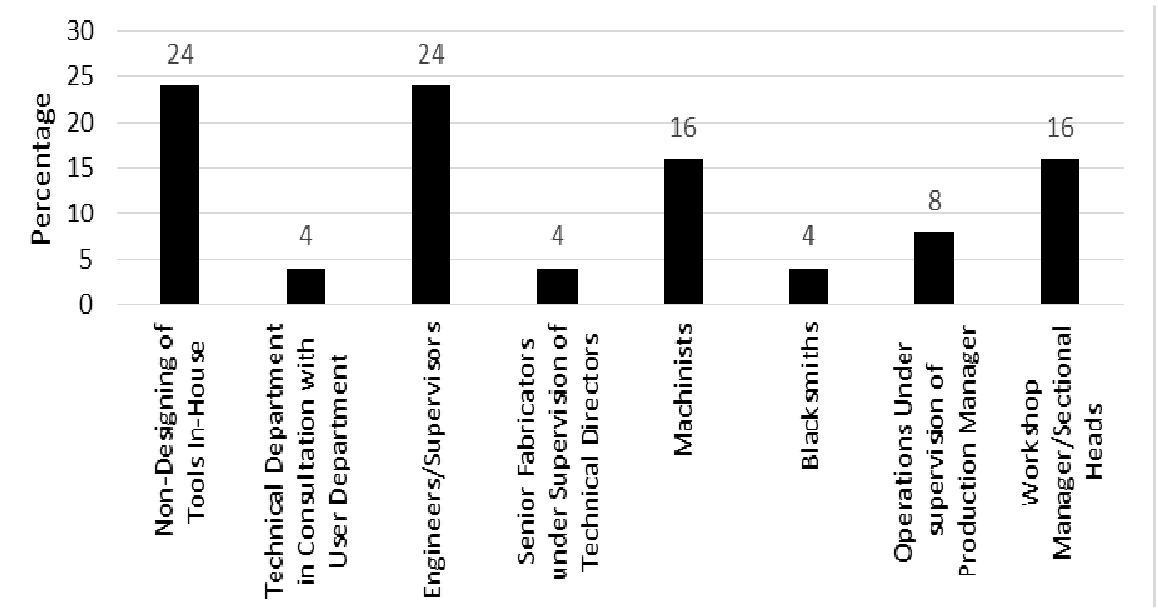

Fig. 3: In-house tool designing responsibility 


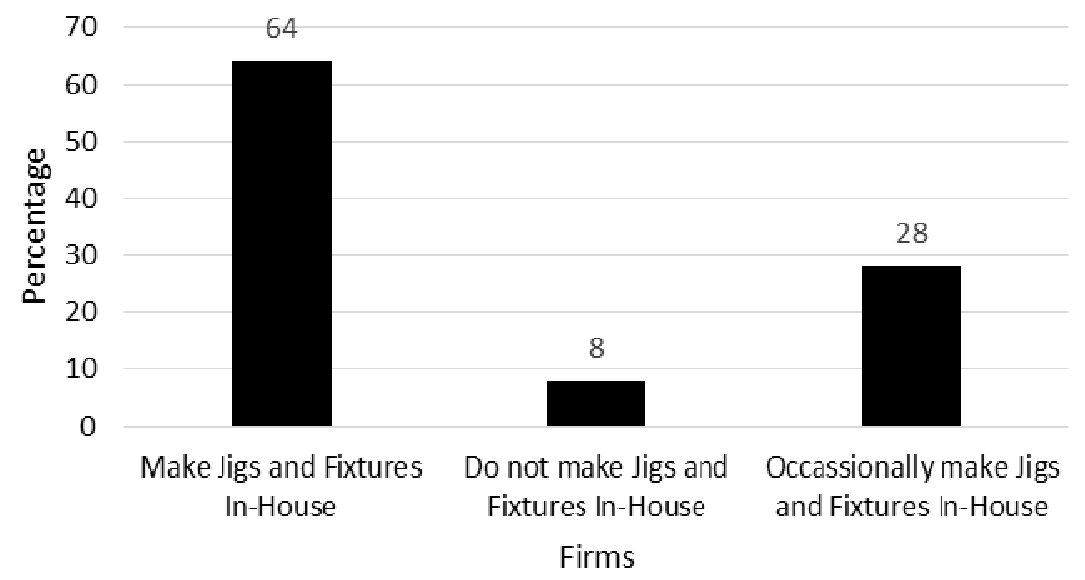

Fig. 4: In-house making of production tooling by firms

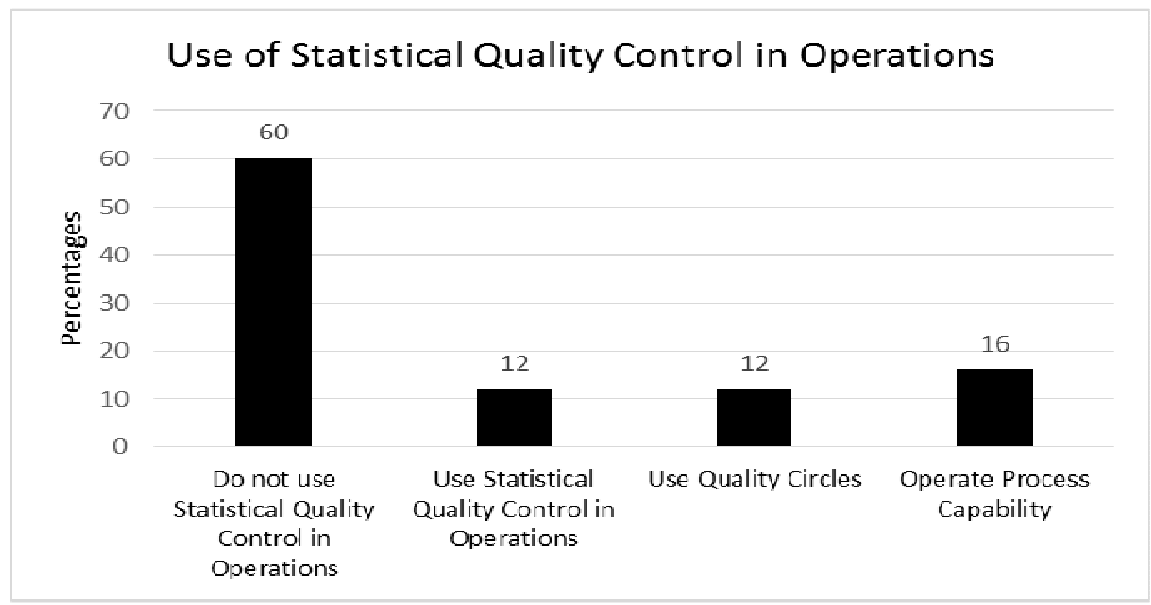

Fig. 5: Use of statistical quality control techniques by companies

\section{Quality control}

Fig. 5 shows that continuous improvement systems such as Quality Circles, Process Capability Studies, and Statistical Process Control (SPC) are being practiced in metal processing industries in Ghana.

The extent of use of statistical process control (SPC) systems is not so encouraging. Only
$12 \%$ do use SPC. Nevertheless it could be concluded that the issue of quality is gaining some attention, albeit slowly, on the shop floor. Responses to the type of continuous improvement system in operation indicate that $60 \%$ of firms use quality circles. Three of the firms out of this percentage undertake process capability studies as well. $16 \%$ perform inspection of raw materials. 
Findings by Amoako-Gyampa and Acquaah (2008), indicate that regardless of the manufacturing strategy adopted by firms in Ghana, quality provides the most benefits with regard to firm performance. From the above discourse it is gratifying to note that some progress is being made in this direction, albeit, slowly.

\section{Benchmarking key world-class ME practices - summary}

Table 2 benchmarks key ME practices in Ghana against world best practice.

It is clear from the foregoing, that leading-edge design/manufacturing technologies, chiefly 3DPrinting and other computer aided design and manufacturing paradigms are increasingly blurring (via automation and integration) the demarcation between the traditional design engineering and manufacturing engineering functions. In these modern systems, widely prevalent in industrialised countries, the manufacturing engineer must know virtually as much about the design processes as he should about the manufacturing realisation of the product itself.

\section{CONCLUSIONS AND RECOMMENDA- TIONS \\ Conclusions}

From the preceding analyses it can be said that the functions of manufacturing engineering are gradually being practiced in the conventional sense to various degrees in metal processing industries in Ghana, and the following conclusions may be drawn:

1. Although production facilities available are somewhat adequate for the current range of products, the benefits missed by Ghanaian manufacturing firms due to the absence of state-of-the-art systems reviewed in section 1.2 means that speed and high product quality and replication needed so much to achieve world-class manufacturing, may not be realised soon.

2. The overwhelming dominance of manual

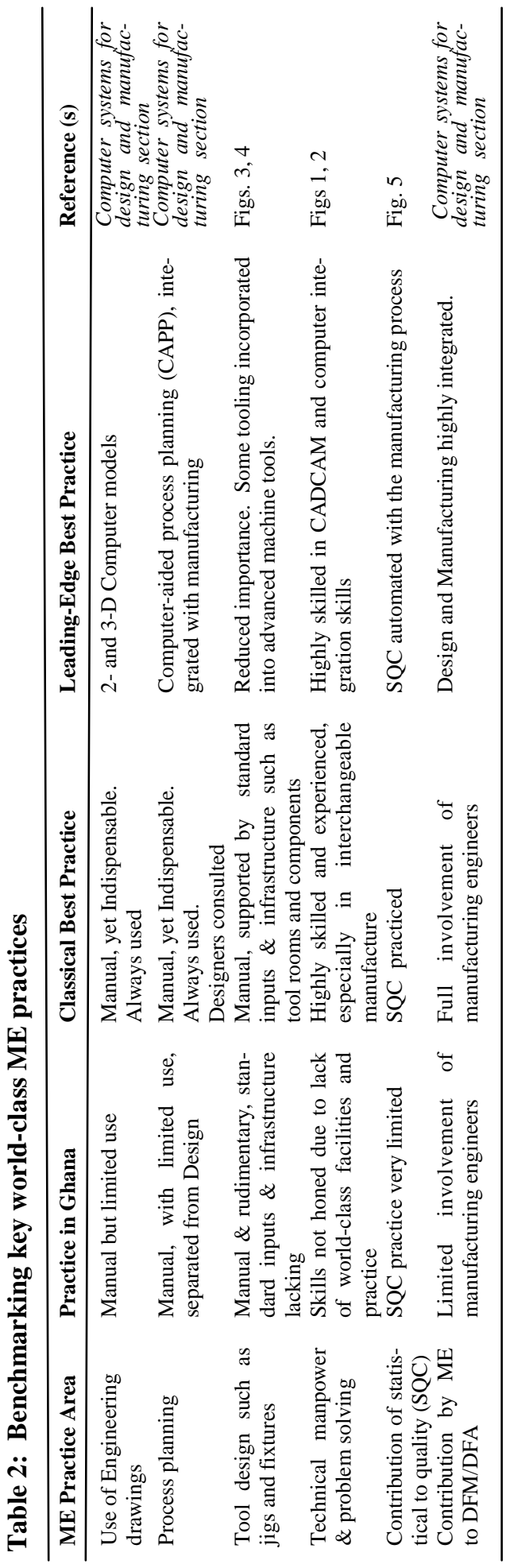

Journal of Science and Technology @ KNUST April 2015 


\section{$50 \quad$ Sackey and Frimpong}

process planning in firms means that benefits of computer-aided process planning (CAPP) such as enhanced product quality, easy and editable documentation, and product innovation potential, are all being missed. This is bad for competitiveness and growth, noting that CAPP eliminates artificial barriers between the traditional product design and manufacturing functions that lead to long lead-times.

3. The rather low promotion of quality control systems for continuous process and product improvement may delay efforts to help Ghana catch up with the newly industrialising countries whose success have depended on export-led growth based on world-class quality products.

4. It appears product innovation is not being actively pursued, judging from the very low rate of new product introduction recorded. However incremental innovations are frequently done. But where manufacturing engineers do offer some contribution to Design for Manufacture, they do not consciously have product innovation in mind as a goal, a disposition considered not to be progressive.

5. Tooling design and development systems are not well developed.

6. On technical manpower, the calibre of staff doing in-house tool design reveals a lack of high quality manufacturing engineers in the sector, perhaps reflecting the lack of relevant specialist training in Ghana.

7. A general lack of technological direction and a manufacturing culture at all levels of Ghanaian society, are among other factors impeding the progress of ME in Ghana.

\section{Recommendations}

The following recommendations are set forth:

1. Industries must set up benchmarking indi- ces so that they can compare their performance against industry leaders.

2. Manufacturing engineering curricula in technical education institutions must be developed. To date there is no institution in Ghana that offers a programme in manufacturing engineering at the tertiary level.

3. Future research could compare Ghana with key developing African countries such as Egypt, Nigeria, or South Africa, and with industrialising countries such as, India, Malaysia and China, for lessons to be drawn.

The implications of the above are that there will be the need for technologically trained people in all aspects of computer-assisted design and manufacturing. Curricula of technical education institutions need to be revamped to reflect current global trends. In striving to achieve efficiencies in its manufacturing, Ghana stands to benefit from countries such as South Korea, Malaysia, and China that have trodden the path of export-led manufacturing success, supported strongly by technical proficiency. As Akira (2004) rightly observed, China achieved remarkable economic growth from 1989 to 1993 on the back of low production costs and rapid acquisition of manufacturing engineering techniques.

\section{REFERENCES}

Akira, K. (2004). Manufacturing: Making a comeback, The Japan Journal, 1(3):18-19.

Amoako-Gyampa, K. and Acquaah, M. (2008). Manufacturing strategy, competitive strategy and firm performance: An empirical study in a developing economy environment, International Journal of Production Economics, 111 (2): 575-592.

Association of Ghana Industries (2011). Association of Ghana Industries (AGI) Membership Register. 
ME practices in the metals sector of Ghanaian industry ... 51

Baldwin, C. Y. and Kim, B. (2000). "Clark Design Rules: The Power of Modularity," MIT Press, Cambridge.

Carnevalli, J. A. and Miguel, P. C. (2008). Review, analysis and classification of the literature on QFD-Types of research, difficulties and benefits. International Journal of Production Economics, 114 (2): 737-754.

Chen, Y., Shir, W. and Shen, C. (2002). Distributed engineering change management for allied concurrent engineering, International Journal of Computer Integrated Manufacturing, 15 (2): 127-151.

Choi, H. G., Ahn, J. O., Jeung, H. S. and Kim, J. S. (2010). A framework for managing risks on concurrent engineering basis, International Journal of Management Science and Engineering Management, 5(1): 44-52.

Deming, W. E. (2000). Out of Crisis. $1^{\text {st }}$ edition. The MIT Press.

Gunasekaran, A. and Yusuf, Y. Y. (2002). Agile manufacturing: a taxonomy of strategic and technological imperatives, International Journal of Production Research, 40 (6): 1357-1385.

Nee, J. G. (2010). Fundamentals of Tool Design, $6^{\text {th }}$ edition. The Society of Manufacturing Engineers, USA
Oppon, C. E., Hackney, P., Shyha, I. and Beeby, J. (2014). Advances in Additive Manufacturing Processes-Conference Proceedings of the Third Applied Research Conference in Africa, 7-9 August 2014, Accra, Ghana p.148-160.

Scallan, P. (2003). Process Planning: The design/manufacture interface. ButterworthHeinemann.

Sheheryar, M. Q. and Chang, W. K. (2014). Analysing the organizational factors of project complexity using structural equation modelling. International Journal of Project Management. In Press, Corrected Proof, Available online 13 May 2014.

Ulrich, K. T. and Ellison, D. J. (2009). Beyond make-buy: internalization and integration of design and production, Production and $\mathrm{Op}$ erations Management, 14(3): 315-330.

Xu, Y., Du, Y., Zeng, Y., Shiming L. (2013), Flexible Manufacturing of Continuous Process Enterprises with Large Scale and Multiple Products, Technology and Investment, 4: 45-56.

Xue-Feng, Y., Lin, Z. and Jie, L. (2013). Smart Analyses and Diagnoses of Piston Product Line Based on Virtual Manufacturing Technology, 6 (18), pp. 3477-3483. 